\title{
Nuclear data for ion beam analysis applications
}

\author{
Paraskevi Dimitriou ${ }^{a}$, Valentina Semkova, and Viktor Zerkin \\ Nuclear Data Section, International Atomic Energy Agency, 1400 Vienna, Austria
}

\begin{abstract}
Nuclear data for Ion Beam Analysis have been compiled and disseminated by the Nuclear Data Section through the Ion Beam Analysis Nuclear Data Library (IBANDL) for over a decade. Recent efforts to enrich IBANDL with gamma-ray producing nuclear reaction cross sections, and to improve search and retrieval features are presented. The coordinated effort to produce reliable evaluated cross-section data for charged-particle reactions for a wider range of applications is also discussed.
\end{abstract}

\section{Introduction}

Ion Beam Analysis is a suite of non-destructive analytical techniques that provide information on the bulk composition and/or depth profile of surface layers of materials. As such they are used in applications ranging from materials analysis to cultural heritage, environmental studies, fusion technologies, biomedical and forensic applications.

For over 10 years, the Nuclear Data Section (NDS) of the IAEA has been serving as the international centre for the collection and dissemination of nuclear data for Ion Beam Analysis. Through a series of Coordinated Research Projects (CRP) [1,2], collaborations with expert scientists and staff efforts, the Ion Beam Analysis Data Library (IBANDL) [3] that contains over 3000 datasets of differential and total cross sections in the low energy region below several $\mathrm{MeV}$ was created and disseminated.

The most recent CRP on Particle-Induced Gammaray Emission spectroscopy (PIGE) [2,4] from 2011 to 2015 has resulted in augmenting the data library by over 300 new datasets of gamma-ray production cross sections needed in the implementation of the PIGE technique in bulk analysis and depth profiling.

Since 2013, the NDS is fully responsible for compilation of data and for maintenance of the retrieval system of IBANDL. Recently, it has also initiated a coordinated effort to evaluate data for charged-particleinduced reactions in the resolved-resonance region in response to emerging needs for such data in a broad spectrum of applications including IBA.

In this paper we present the current status of the content of IBANDL, as well as the new retrieval features that have been introduced to facilitate the user in searching, retrieving and plotting data. We report on the progress made in the coordinated evaluation effort and finally, we discuss the perspectives for further development in the future.

\section{Coordinated research project on PIGE}

\subsection{Objectives}

The main goal of the CRP on PIGE (2011-2015) $[2,3]$ was to create a data library for IBA that contains reliable and usable data on charged-particle gamma-ray cross sections that will be made freely available to the user community. To achieve this goal, the most important nuclear reactions for the PIGE technique were identified, followed by a thorough search in the literature and other electronic databases (EXFOR [5], NACRE [6]) for relevant data. Data from different sources were compared and new measurements were carried out either when no data were found or when the data were discrepant. About 150 new data sets were measured during the CRP and almost 200 were compiled from the existing literature as can be seen in Fig. 1.

\subsection{Coordinated differential cross-section measurements}

To obtain a consistent set of measured data for comparison and eventually for evaluation, a certain methodology was adopted by all the laboratories involved in the measurements. To identify possible sources of discrepancies and systematic errors, a coordinated measurement exercise was first conducted. Ten laboratories measured the $844 \mathrm{keV}$ gamma-line from the ${ }^{27} \mathrm{Al}\left(\mathrm{p}, \mathrm{p} \gamma_{1-0}\right)^{27} \mathrm{Al}$ reaction in a given energy range and at given energy intervals. Some of the results of the exercise are shown in Fig. 2.

One of the interesting findings of this inter-comparison exercise was the inconsistency of the energies of the narrow resonances used for accelerator energy calibration, mainly at energies above $2 \mathrm{MeV}$. Clearly, an effort is needed to improve the situation. The reported cross-section data overall agree within the quoted uncertainties, which on average are of the order of $7 \%$.

\subsection{Thick target yields}

Measurements of thick target yields are useful both for extracting resonance parameters and for bulk analysis relative to standards. In the context of this CRP, thick

\footnotetext{
a e-mail: p.dimitriou@iaea.org
}

(C) The Authors, published by EDP Sciences. This is an Open Access article distributed under the terms of the Creative Commons Attribution License 4.0 (http://creativecommons.org/licenses/by/4.0/). 


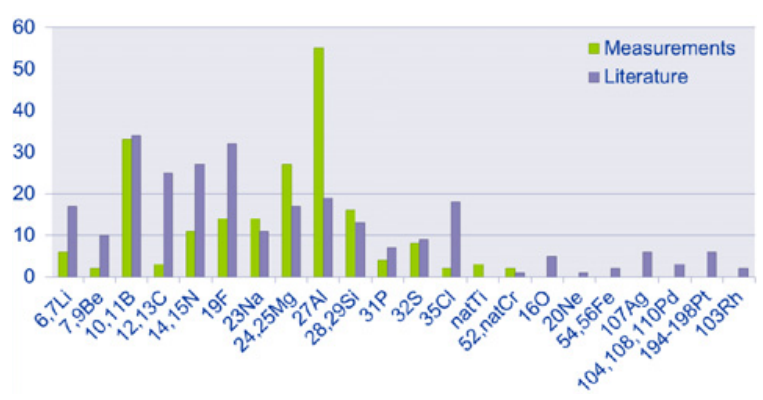

Figure 1. Distribution of data measured or found in the literature over the elements used in PIGE applications.

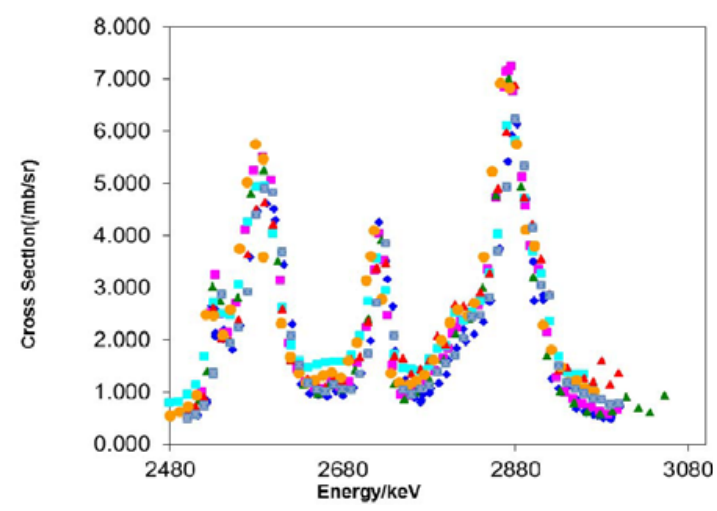

Figure 2. Comparison of cross sections for the $844 \mathrm{keV}$ gamma ray emitted from the ${ }^{27} \mathrm{Al}\left(\mathrm{p}, \mathrm{p} \gamma_{1-0}\right)^{27} \mathrm{Al}$ reaction measured by seven CRP laboratories at different angles. Although the distribution of the gamma ray is isotropic, the cross sections are given per ( $\mathrm{sr}$ ) to account for the solid angle of the gamma detector.

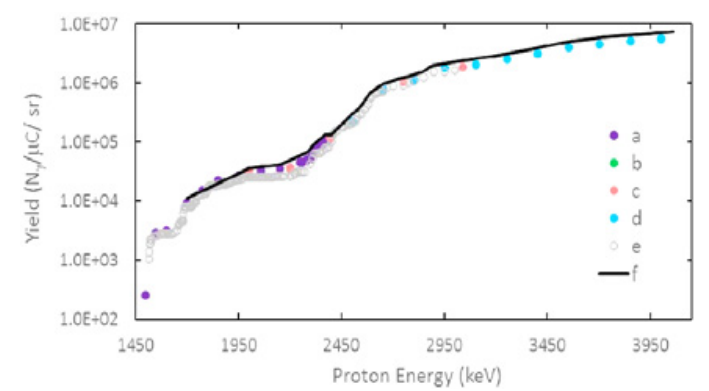

Figure 3. Comparison of thick target yields corresponding to the $844 \mathrm{keV}$ gamma line emitted by a pure $\mathrm{Al}$ target, measured at different angles in the PIGE CRP (see text). Although the distribution of the gamma ray is isotropic, the yields are given per (sr) to account for the solid angle of the gamma detector.

target yields served as benchmarks to test the measured differential cross section data. In Fig. 3, five different measurements of thick target yields for the $844 \mathrm{keV}$ gamma-line from the ${ }^{27} \mathrm{Al}\left(\mathrm{p}, \mathrm{p} \gamma_{1-0}\right)^{27} \mathrm{Al}$ reaction covering an energy range from 1.5 to $4 \mathrm{MeV}$ are compared with calculated yields. Similar comparisons have been made for all measured differential cross sections and the results will be published in the CRP Technical Report which is in preparation.

The importance of benchmark thick target yield measurements to validate cross section data was discussed extensively at an IAEA Technical Meeting and the conclusions can be found in Ref. [7].

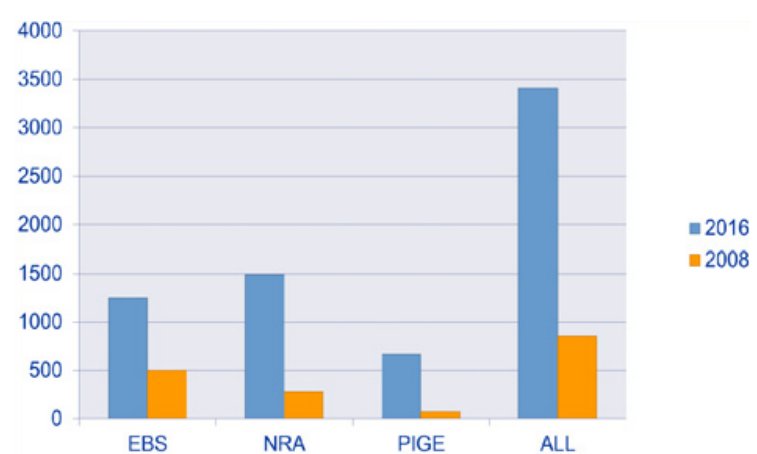

Figure 4. Number of datasets for EBS, NRA and PIGE reactions available in IBANDL.

\subsection{PIGE analysis code}

The calculated yields (thick solid line) shown in Fig. 3 were obtained by integrating the measured differential cross sections using the ERYA code [8]. In particular, two different sets of measurements covering two different energy ranges (one up to $3 \mathrm{MeV}$ shown in Fig. 2 and another from 3 to $4 \mathrm{MeV}$ ) were combined in the integration process.

The development of the ERYA code was another objective of the PIGE CRP: using the cross section and stopping power databases, it calculates the material composition in the case of bulk analysis, and the layer composition in the case of depth analysis, thus enabling standardless PIGE analyses.

The ERYA code is freely distributed, however, in the future an online calculation tool that would allow the user to directly calculate thick target yields on-the-fly based on a selection of cross-section data and material composition will also be possible from the IBANDL interface.

\section{Ion Beam Analysis Nuclear Data Library (IBANDL)}

\subsection{Content}

IBANDL currently contains 3640 datasets of nuclear reaction cross sections and yields, for 182 charged-particle reactions on 99 different targets. The datasets include differential cross sections and thick-target yields measured at various angles, as well as total cross sections (excitation functions), and are relevant for IBA techniques such as Elastic Backscattering (EBS), Nuclear Reaction Analysis (NRA) and PIGE. In addition, the retrieval interface allows access to and comparison with the limited list of 45 evaluated cross-sections available at the SigmaCalc web site [9].

The progress in uploading new datasets and maintaining IBANDL up-to-date over the past decade is shown in Fig. 4.

\subsection{New features}

Efforts are continuously being made to improve the retrieval interface of IBANDL to meet user's needs and requests. The most recent features added are:

- Links to EXFOR database when the cross-section data or other relevant data of the same author are available there 

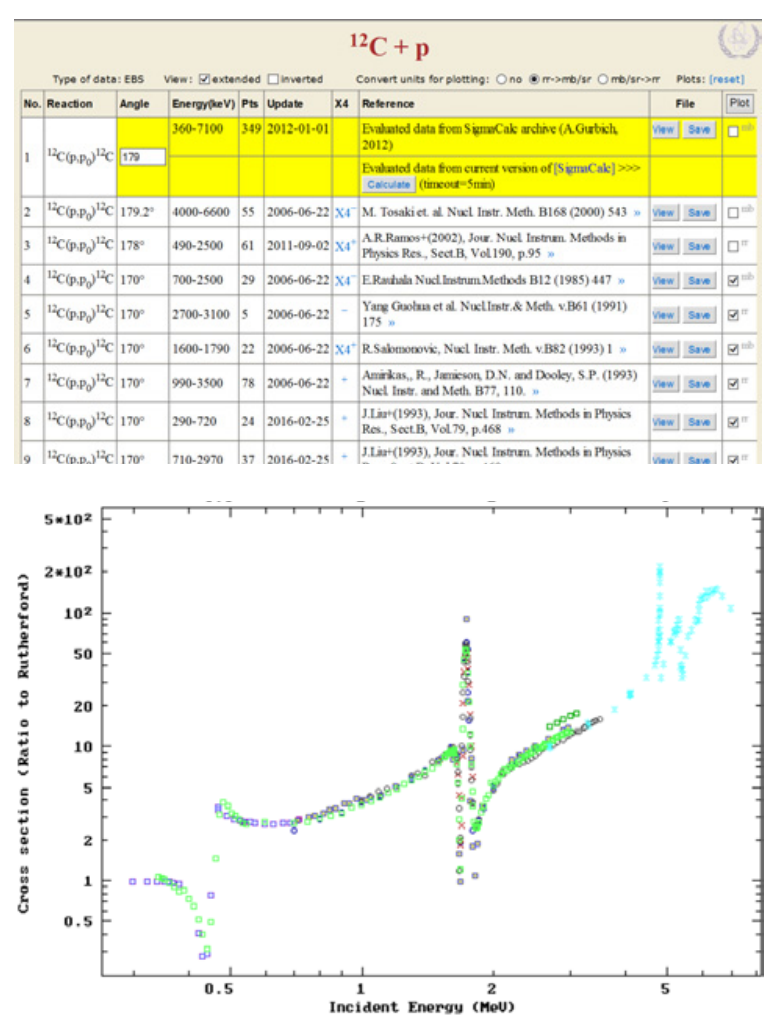

Figure 5. Example of converting ratio-to-Rutherford data to absolute cross section data and plotting for ${ }^{12} \mathrm{C}\left(\mathrm{p}, \mathrm{p}_{0}\right)^{12} \mathrm{C}$ at angle $170^{\circ}$ in IBANDL [3].

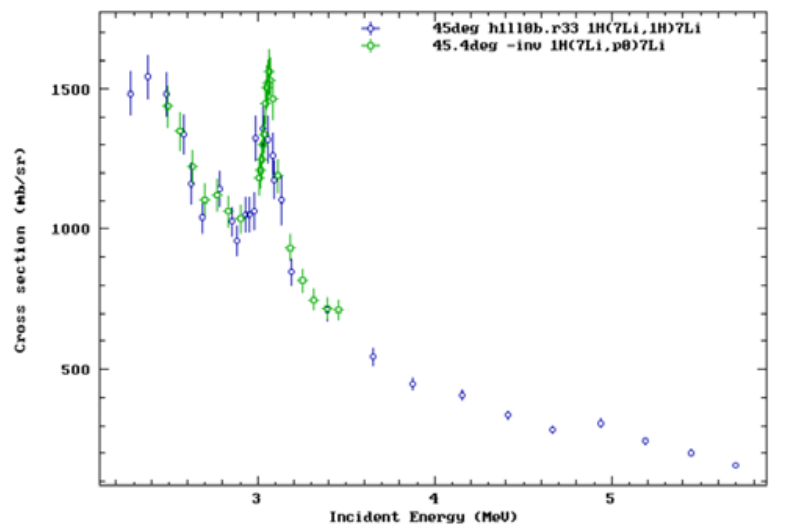

Figure 6. Cross sections for the reaction ${ }^{1} \mathrm{H}\left({ }^{7} \mathrm{Li}, \mathrm{p}\right)^{7} \mathrm{Li}[10]$ compared with the inverted cross sections for proton elastic scattering by ${ }^{7} \mathrm{Li}[11]$ in IBANDL [3].

- Summary tables of the available data for the different IBA techniques (EBS, NRA, PIGE) and for all of them together

- Conversion of cross-section data from ratio-toRutherford units to absolute cross-section units ( $\mathrm{mb} / \mathrm{sr}$ ) and vice-versa and graphical comparison of the results (see Fig. 5)

- Transformation of cross-section data from forward kinematics to inverse kinematics and comparison of results in graphical form (see Fig. 6). The transformed cross sections are also tabulated

- Uploading and graphical comparison of user's data files (in R33 format) with existing IBANDL data.

An example of the transformation to inverse kinematics is shown in Fig. 6. This feature is particularly useful in hydrogen profiling applications where very often the cross sections for the reaction of a heavy projectile on hydrogen atoms are unknown. In such cases one can use the cross sections for elastic scattering of protons on the heavy target which are more likely to have been measured, by simply converting them to inverse kinematics by a simple press of a button on IBANDL.

\section{Evaluation of charged-particle induced reactions in the resolved resonance region}

For many years the IBA community has been relying on one single source of evaluated differential cross sections: the SigmaCalc [9] online calculator. On the other hand, the widely used evaluated data files are incomplete as far as charged-particle induced reactions in the resolved resonance region are concerned.

However, recent developments in existing and emerging applications clearly indicate the need for reliable charged-particle induced reactions at low energies. A Consultant's Meeting of R-matrix code developers was held at the IAEA from 7 to 9 December 2015 [12] to discuss the capabilities and specific features of six different R-matrix codes, namely, SAMMY, AZURE2, FRESCO, AMUR, EDA, and HYRMA (see abovementioned summary report for references), that are currently used to perform R-matrix fits of charged-particleinduced reactions in the resolved resonance region.

A condition to perform useful comparisons of R-matrix calculations is the translatability of R-matrix input and output parameters between the various codes. As a result, a code was developed to convert R-matrix fits between several formats, including ENDF-6 [13], GND [14], and the various formats used for the input and output of the above-mentioned R-matrix codes.

The next step is to perform a joint evaluation, to establish the methodology, treatment of uncertainties and correlations. In the meantime, efforts are ongoing to make existing evaluations of charged-particle reactions in the resolved resonance region available to the user community through ENDF [15] and IBANDL [3].

\section{References}

[1] Development of a Reference Database for Ion Beam Analysis, IAEA-TECDOC-1780, Vienna 2015. (http://www-nds.iaea.org/publications/ tecdocs/TE-1780_web.pdf)

[2] P. Dimitriou, et al., Nucl. Instr. and Meth. B 317, 33-36 (2016); 10.1016/j.nimb.2015.09.052

[3] Ion Beam Analysis Nuclear Data Library (IBANDL), URL: http://www-nds.iaea.org/ibandl/

[4] P. Dimitriou, A.P. Jesus, in 3rd RCM of CRP on Particle-Induced Gamma-ray Emission spectroscopy (PIGE), 7-11 April 2014, IAEA, INDC(NDS)-0664 (http://www-nds.iaea.org/publications/ indc/indc-nds-0664.pdf)

[5] N. Otuka, et al, Nuclear Data Sheets 120, 272 (2014); URL: http: //www-nds.iaea.org/exfor/

[6] C. Angulo, et al., Nucl. Phys. A 656, 3-183 (1999)

[7] M. Chiari, P. Dimitriou, in Summary Report of IAEA Technical Meeting on Benchmarking 
Experiments for Ion Beam Analysis, 26-29 May 2015, INDC(NDS)-0690 (https : //www-nds . iaea.org/publications/indc/indc-nds0690.pdf)

[8] M. Fonseca, et al., Nucl. Instr. and Meth. B 268, 1806 (2010)

[9] SigmaCalc Online Calculator, URL: http:// sigmacalc.iate.obninsk.ru/

[10] Z. Siketic et al., Nucl. Instr. and Meth. B 229, 180 (2005)

[11] W.D. Warters, W.A. Fowler and C.C. Lauristen Phys. Rev. 91, 917 (1953)

[12] P. Dimitriou, R. deBoer, S. Kunieda, M. Paris, I. Thompson, A. Trkov, in Summary Report of IAEA Consultant's Meeting on R-matrix Codes for Charged-particle Reactions in the Resolved-Resonance Region, 7-9 December 2016, IAEA, INDC(NDS)-703 (https://www-nds . iaea.org/publications/indc/indc-nds-0703. pdf)

[13] ENDF-6 Formats Manual, Eds. A. Trkov, M. Herman and D.A. Brown, CSEWG Document ENDF-102, Report BNL-90365-2009 Rev.2, December 2011

[14] Generalized Nuclear Data, (GND), OECD/WPEC Subgroup 38, URL: https://www.oecd-nea. org/science/wpec/sg38/

[15] Evaluated Nuclear Data File (ENDF), URL: http://www-nds.iaea.org/endf / 